Ann. Biol. anim. Bioch. Biophys., I972, 12 (2), 335-339.

\title{
INFLUENGE DE DIFFÉRENTES FRACTIONS PRÉPARÉES A PARTIR D'HUILE DE SOJA CHAUFFÉE SUR LE MÉTABOLISME DE LA THIAMINE ET DE LA RIBOFLAVINE (CHEZ LE RAT)
}

\author{
M. LHUISSIER et B. POTTEAU \\ avec la collaboration technique \\ de Françoise Nivet, Geneviève Chandellter et W. Loisel \\ Station de Recherches sur les Aliments de l'Homme, \\ Centre de Recherches de Dijon, I. N.R. A., \\ 21 - Dijon
}

Dans un précédent travail, nous avons étudié les effets d'huiles thermopolymérisées, n'ayant fait l'objet d'aucun fractionnement, sur la teneur du foie en quelques vitanines du groupe $B$ (LhUissier et Potteau, 197r). Nous avons alors signalé un phénomène d'augmentation des concentrations vitaminiques hépatiques, précédée d'une diminution du rejet urinaire de la thiamine et de la riboflavine.

Dans la présente étude, nous avons cherché le type de composé formé au cours du chauffage des huiles qui peut être responsable de ces effets.

L'huile de soja raffinée témoin a la composition en acides gras suivante (en p. Ioo) : acide palmitique $(\mathrm{I} 6: \mathrm{o})$ et palmitoléique $(\mathrm{r} 6: \mathrm{I})=\mathrm{ro}, 0 ;$ acide stéarique $(\mathrm{I} 8: 0)=3,7 ;$ acide oléique $(\mathrm{I} 8: \mathrm{I})=24,0$; acide linoléique $(\mathrm{I} 8: 2)=5 \mathrm{I}, 5$; acide linolénique $(\mathrm{I} 8: 3)=8,0$; acide arachidique $(20: 0)=0,9$; acide béhénique $(22: 0)$ et érucique $(22: \mathrm{I})=1,7$. d'azote.

Cette huile est thermopol ymérisée par chauffage à $275^{\circ} \mathrm{C}$, pendant $\mathrm{I} 2$ heures, avec barbotage

Le mode de préparation des fractions a été dếcrit dans une publication antérieure (РotTEAU et al., 1970). Le tableau I rappelle le mode de préparation des fractions issues de l'huile chauffée. Par rapport aux acides gras initiaux, le bilan global moyen a été le suivant:

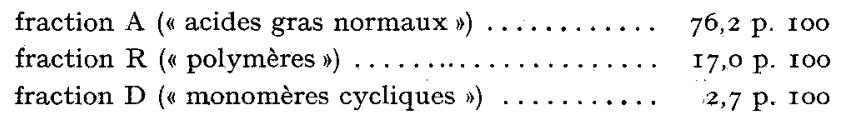

Les fractions $\mathrm{D}_{2}(4,0 \mathrm{p}$. IOO) et $\mathrm{R} 2$ (inférieure à o.I p. IOo) se sont révélées être constituées d'acides gras normaux.

Nous utilisons le même régime qu'au cours des essais précédents (Potreau et al., 1970); il contient $4 \mathrm{mg}$ de thiamine et $4 \mathrm{mg}$ de riboflavine par $\mathrm{kg}$.

La quantité globale de l'ensemble lipides + saccharose étant maintenue constante $(4 \circ \mathrm{p} .100$ du régime), l'apport lipidique est présenté sur le tableau 2, détermininaṇt 9 types de régimes correspondant à 9 lots.

Les 90 rats de l'expérience sont soumis à ces régimes dès l'âge de 5 semaines. Ils pèsent alors, 


\section{TABLEAU I}

Schéma de préparation des diverses fractions à partir de l'huile de soja chauffée (D'après Potteau et al., 1970)

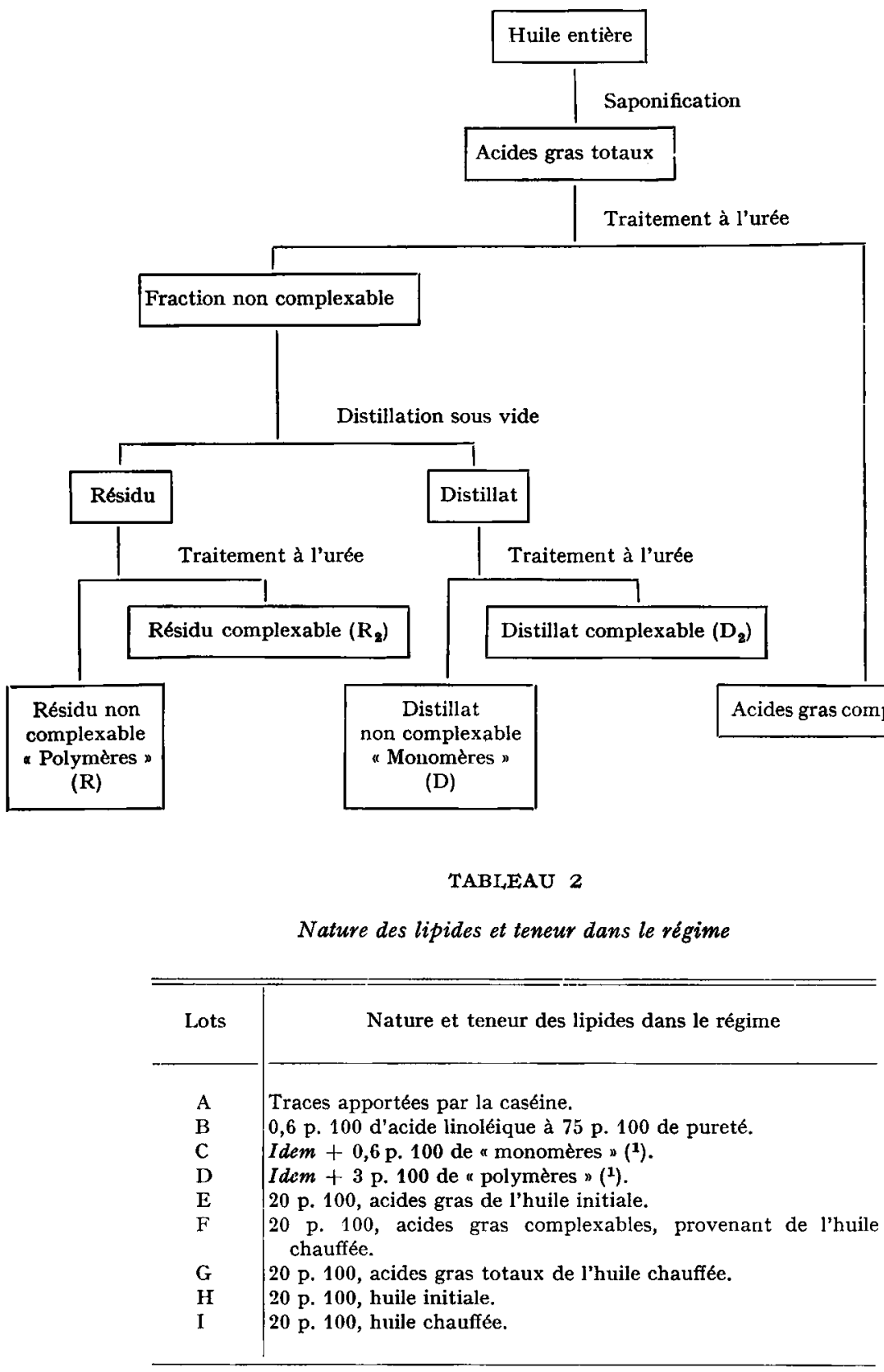

(1) Ces fractions sont introduites dans $100 \mathrm{~g}$ de régime en quantités voisines de celles extraites à partir de $20 \mathrm{~g}$ des acides de l'huile chauffée. 
en moyenne, $95 \mathrm{~g}$. Trois jours plus tard et pendant Io jours, 5 rats de chaque lot sont placés dans des dispositifs de bilans, pour la récolte des urines. Après avoir été soumis pendant 3 mois au régime expérimental, les animaux sont sacrifiés et leur foie est prélevé pour le dosage de l'azote, du phosphore, de la thiamine, de la riboflavine et des lipides totaux.

La thiamine et la riboflavine sont déterminées par méthode microbiologique en utilisant respectivement Lactobacillus viridescens et Lactobacillus casei cultivés sur milieux Difco appropriés. L'azote et le phosphore sont dosés par méthodes colorimétriques à l'autoanalyseur "Technicon ", en utilisant respectivement la réaction de Berthelot (formation d'un complexe bleu d'indophénol lu à $630 \mathrm{~m} \mu$ ) et la formation d'un complexe phosphovanadomolybdique non réduit lu à $420 \mathrm{~m} \mu$ (réaction de Misson).

L'évolution pondérale des animaux des lots, A, B, C, D, H et I est voisine. Celle des animaux des lots $E, F$ et $G$ est inférieure.

Les analyses urinaires (fig. I et 2) mettent en évidence la responsabilité de la fraction "monomères " (lot C), dont l'administration entraîne l'abaissement simultané des excrétions de la thiamine et de la riboflavine, par rapport à celles du lot $B$, abaissement qui est analogue à celui que l'on observe lorsque l'huile chauffée est substituée à l'huile fraîche (comparaison lot $\mathrm{I} / \mathrm{lot} \mathrm{H}$ ).

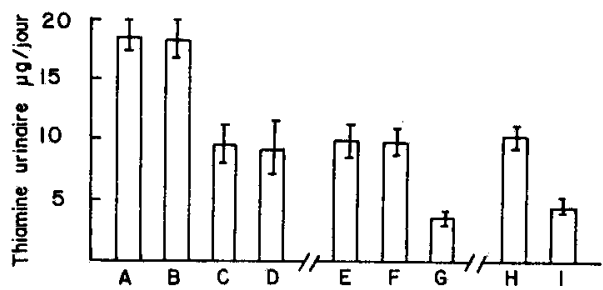

FIG. I. - Eliminations urinaires de thiamine mesurées au cours de la période de mise en bilan (résultats exprimés en $\mu \mathrm{g} /$ jour, 5 animaux par lot, moyennes et erreurs standards)

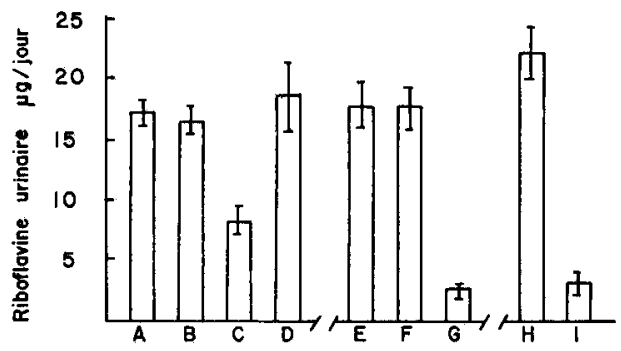

Frg. 2. - Eliminations urinaires de riboflavine mesurếes au cours de la période de mise en bilan

(résultats exprimés en $\mu \mathrm{g} / \mathrm{jour}, 5$ animaux par lot, moyennes et erreurs standards)

L'administration de la fraction "polymères " (lot D) abaisse l'élimination urinaire de la thiamine mais ne modifie pas celle de la riboflavine.

Les animaux ne présentent pas de phénomène de stéatose (tabl. 3). L'hypertrophie hépatique relative est à noter dans les lots $\mathrm{C}, \mathrm{D}, \mathrm{G}$ et $\mathrm{I}$.

On observe des quantités accrues de thiamine et de riboflavine dans le foie des animaux qui ingèrent les huiles chauffées. L'augmentation des concentrations est significative : elle atteint 9 et 25 p. roo, respectivement. En revanche, aucune des fractions ne modifie significativement les taux vitaminiques.

Aux valeurs les plus fortes des concentrations en riboflavine du foie correspondent les rejets urinaires de la même vitamine les plus faibles. 


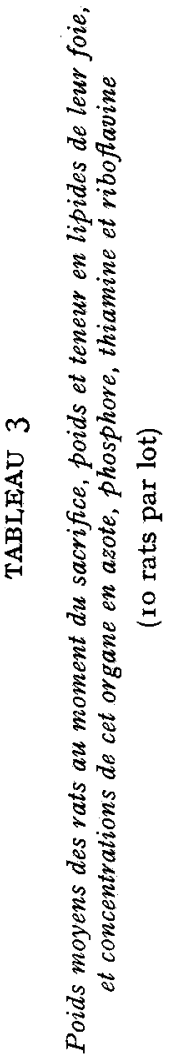

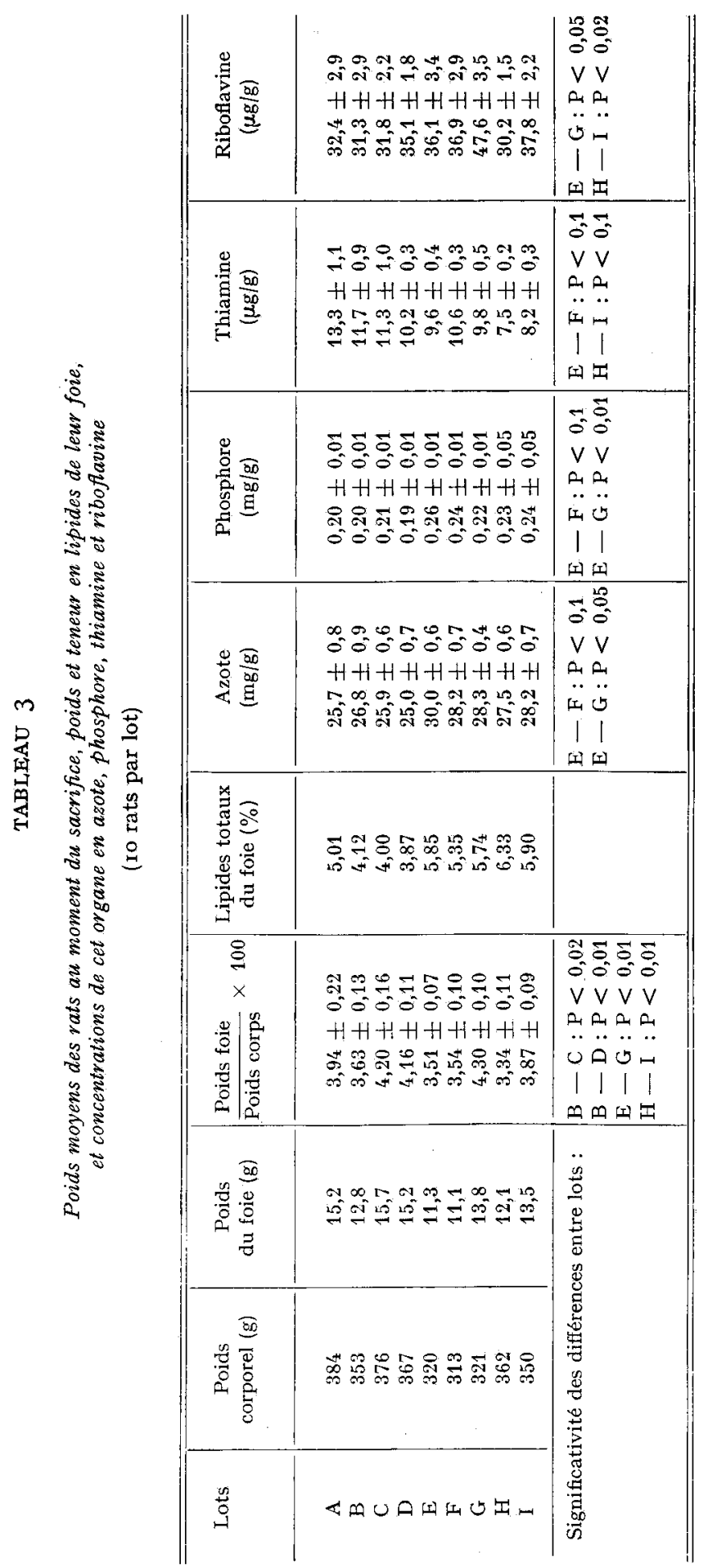


Au début de l'expérience, la fraction "monomères " apparait responsable de la diminution du rejet urinaire des deux vitamines, et la fraction "polymères " responsable de la diminution du rejet urinaire de la thiamine. En revanche, les déterminations effectuées au niveau du foie, après 3 mois d'administration des régimes expérimentaux, ne permettent pas d'être aussi catégorique. A ce stade, on ne peut que mettre en évidence, de façon significative, les augmentations des concentrations de la thiamine et de la riboflavine au niveau du foie chez les animaux ingérant l'huile chauffée entière. Si l'on tient compte de l'augmentation de la masse hépatique par rapport au poids corporel, les différences observées sont encore plus marquées.

L'expérience elle-même ne suggère aucune explication précise en ce qui concerne le mécanisme des faits observés. Toutefois, on peut envisager l'hypothèse de la synthèse d'un inducteur des systèmes enzymatiques métabolisant les "drogues " dans l'huile chauffée, inducteur qui serait surtout concentré dans la fraction "monomères " mais dont tous les effets se feraient sentir dans l'huile chauffée entière (ou avec l'ensemble des acides gras provenant de celle-ci).

Ainsi s'expliquerait l'accumulation de vitamines coenzymatiques au niveau du foie, légèrement hypertrophié, des animaux recevant, soit l'huile chauffée entière, soit l'ensemble des acides gras provenant de celle-ci. Cet effet ne serait pas mis en évidence par les dosages de l'azote et du phosphore total du foie car ce phénomène d'induction n'intéresserait qu'une fraction seulement de la cellule hépatique (fraction microsomiale).

Les produits du chauffage de l'huile concentrés dans la fraction "monomères " (acides gras cyclisés) semblent être, d'après les tests urinaires, les principaux responsables des effets des huiles chauffées entières vis-à-vis des rejets diminués des deux vitamines dosées. Les dosages tissulaires pratiqués au niveau du foie ne permettent pas la même démonstration : seule l'huile chauffée entière entraîne une élévation significative (seuil de significativité $\mathrm{P}<0,02$, dans le cas de la riboflavine) des teneurs vitaminiques exprimées en concentrations.

Reçu pour publication en octobre 1971.

\section{SUMMARY}

\section{EFFECTS OF SOME EXTRACTED FRACTIONS \\ OF HEATED SOYBEAN OIL, ON THIAMINE AND RIBOFLAVIN METABOLISM}

Five-week-old wistar rats were fed semi-synthetic diets containing fresh soybean oil, soybean oil heated to $275^{\circ} \mathrm{C}$ for 12 hours under nitrogen, or extracted fractions of heated soybean oil. Urinary excretions of thiamine and riboflavin were measured at the beginning of the experiment. The content of these vitamins was measured in the liver at the end of the experiment as well as that of nitrogen, phosphorus, and lipids.

When heated soybean oil is given to the rats, urinary excretions of B vitamins are significantly decreased, while their content in the liver is increased. Urinary excretions of these vitamins are also decreased when the "monomeric " fraction of the heated oil is added to the diet.

\section{RÉFÉRENCES BIBLIOGRAPHIQUES}

Lhuissier M., Potreau B., I97I. Influence des huiles de soja et de lin thermopolymérisées sur la teneur du foie en thiamine, riboflavine, vitamine $\mathrm{B}_{\mathrm{s}}$ et niacine (chez le Rat). Ann. Nutrit. Alim., 25, 2I5-22I.

Potteau B., Lhuissier M., Leclerc J., Custot F., Mezonnet R., Cluzan R., ig7o. Recherches sur la composition et les effet physiologiques de l'huile de soja chauffée et de différentes fractions obtenues à partir de cette huile. Rev. fr. Corps Gras. 17, 143-163, 235-245. 